suggesting total reliance on carers for all of their needs. This disability was not accounted for by those in care homes having more medical morbidity as we had hypothesised. Rather, the combination of deficit symptoms and poor cognition appeared to be responsible. Poor cognition with or without dementia is reported in older people with schizophrenia (Harvey et al, 1999). Thus the disablement of care home residents seems likely to be intrinsic to their psychotic disorder, although the effects of long-term institutionalisation cannot be ruled out as an additional factor.

There exists no national service framework that would adequately address the needs of older people with psychosis (Royal College of Psychiatrists, 2002). The two probably most relevant here are not directly concerned with this group - the National Service Framework for Older People (Department of Health, 2001) focused on dementia and depression, and the National Service Framework for Mental Health (Department of Health, 1999) on adults of working age. Considering the complex handicaps arising from their disorder, detailed care planning and review by specialist psychiatric services are imperative for older people with schizophrenia residing in care homes. Despite this, few of those in care homes that took part in this study received input from any member of the Old Age Psychiatry Community Mental Health Team other than the consultant, whereas a majority of those in their own homes received some input from those services. It is not known whether this is typical of other services or not, but it warrants further research. What also need to be examined are the pathways of entry into care homes among this population group.

\section{Declaration of interest}

None.

\section{References}

BURVILL, P.W, MOWRY, B. \& HALL,W. D. Advances in Psychiatric Treat, 10, (1990) Quantification of physical illness 27-34.

in psychiatric research in the elderly.

International Journal of Geriatric

Psychiatry, 5, 161-170.

DEPARTMENT OF HEALTH (1999)

National Service Framework for Mental

Health: Modern Standards and Service

Models. Department of Health.

DEPARTMENT OF HEALTH (2001)

National Service Framework for Older

People. Department of Health.

FOLSTEIN, M. F., FOLSTEIN, S. E. \&

MCHUGH, P. R. (1975) 'Mini-Mental

State': a practical method for grading

the cognitive state of patients for the

clinician. Journal of Psychiatric

Research, 12, 185-198.

HARVEY, P. D., STERMAN, J. M., RICHARD, C., et al (1999) Cognitive decline in later life schizophrenia: A longitudinal study of geriatric

chronically hospitalised patients.

Biological Psychiatry, 45, 32-40

JOLLEY, D., KOSKY, N. \& HOLLOWAY, F. (2004) Older people with longstanding mental illness: the graduates.

KAY, S. R., FISZBEIN, A.\& OPLER, L. A

(1987) The Positive and Negative Syndrome Scale for schizophrenia. Schizophrenia Bulletin, 13, 261-276.

LAWTON, M. P. \& BRODY, E. M. (1969) Assessment of older people: Selfmaintaining and instrumental activities of daily living. Gerontologist, 9, 179186.

MCNULTY, S.V., DUNCAN, L., SEMPLE, M., et al (2003) Care needs of elderly people with schizophrenia. British Journal of Psychiatry, 182, 241-247.

ROYAL COLLEGE OF PSYCHIATRISTS (2002) Caring for People who Enter Old Age with Enduring or Relapsing Mental Illness. College Report CR110, Royal College of Psychiatrists.

WOODS, S.W. (2003) Chlorpromazine equivalent doses for the newer atypical antipsychotics. Journal of Clinical Psychiatry, 64, 663-667.

WORLD HEALTH ORGANIZATION (1993) The ICD-10 Classification of Mental and Behavioural Disorders. WHO.

Emily Clancy 4th Year Medical Student, University of Manchester, *Robert C. Baldwin Department of Old Age Psychiatry, Edale House, Manchester Mental Health and Social Care NHS Trust, Manchester Royal Infirmary, Oxford Road, Manchester M13 9BX, UK, email: robert.c.baldwin@ manchester.ac.uk

WAQQAS AHMAD KHOKHAR, IMRAN HAMEED, MOHAMMED MUBASHIR ALI,

JAVARIA SADIQ AND PETER BOWIE

\title{
To trust or not to trust? Faith issues in psychopharmacological prescribing
}

\section{AIMS AND METHOD}

To ascertain attitudes, awareness, knowledge and variations in prescribing habits of psychiatrists when addressing issues of faith, culture and dietary requirements (in keeping with the General Medical Council's and the Royal College of Psychiatrists' guidelines), and how these may affect the trust in doctorpatient relations. We undertook a cross-sectional postal survey aimed at clinicians in South West Yorkshire Mental HealthTrust.

RESULTS

We obtained 38 responses $(40 \%$ of the total number of questionnaires sent out) from two mailshots. Although the majority of respondents indicated that they were aware of the presence of potentially forbidden animal-derived ingredients in medication, only half expressed the view that a discussion regarding the above should take place prior to prescribing such medication.

\section{CLINICAL IMPLICATIONS}

Diversity training combined with taking a genuine interest in patients' wishes will not only minimise 'mistrust' but also facilitate prescribing in a therapeutic relationship.
'Patients must be able to trust doctors with their lives and well being. To establish that trust doctors must respect patient's autonomy and patients must be given sufficient information in a way that they can understand and are able to exercise their right to make informed decisions about their care. For example, patients may need more information to 


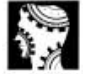

original papers make an informed decision about a procedure, investigation or a condition which, if present, could have serious implications for the patient's employment, social or personal life. When providing information, doctors must do their best to learn about their patient's individual needs and priorities. For example, patient's beliefs, culture, occupation or other factors may have a bearing on the information they need in order to reach a decision. Doctors must not make assumptions about patient's views, but discuss these matters with them and ask them whether they have any concerns. Doctors should provide patients with appropriate information which should include an explanation of any risks to which they may attach particular significance. Doctors should not withhold information necessary for decision making unless they judge that disclosure of such relevant information would cause the patient serious harm. In this context, serious harm does not mean that the patient would become upset or decide to refuse treatment' (General Medical Council, 1998).

Medication is probably the most frequently used form of treatment prescribed by clinicians within the mental health setting. Non-adherence to pharmacotherapy in mental illness significantly impacts upon the lives and well-being of people with mental health problems (Patel \& David, 2005). The causes of poor adherence to treatment are numerous (Robinson et al, 1999) but beliefs regarding mental illness and psychotropic medication are significant factors cited in the literature (Hughes et al, 1997). Although it is generally thought that religious beliefs do not influence medication compliance (Dein, 2004), resolving non-adherence to pharmacotherapy should involve a comprehensive assessment of the patient's demographics, social circumstances, and cultural and religious beliefs (Sattar et al, 2004a; Subach \& Abdul-Ezz, 1999). Some researchers claim that not only do patients with strong spiritual values cope better with mental illness but their understanding of illness behaviour is also greater, giving them better insight into their condition (Kirov et al, 1998). Such factors directly impact upon treatment compliance and engagement in therapy (Batson \& Ventis, 1993; Koenig, 2001; Pargament \& Brant, 1998).

Religious laws do not restrict the use of psychotropic medications but many do forbid the use of animal-based derivatives, specifically gelatinous products and stearic acid which are generally derived from beef and/or pork. This has major implications for many patients, particularly the followers of Judaism, Islam, Hinduism, Buddhism, Seventh Day Adventism and the Christian Orthodox Church (Singsit et al, 2000; Leung et al, 2005). There are also over 4 million vegetarians in the UK and the number is ever growing (Food Standards Agency, 2005).

'All prescribers have an obligation to respect their patient's medical, cultural and religious dietary requirements (e.g. gluten-free for patients with coeliac disease, avoidance of animal gelatin for vegetarian, Jewish or Muslim patients)' (Royal College of Psychiatrists, 2004).

Lack of consideration for these matters will negatively affect the doctor-patient relationship. Although research in this important area has been scarce (Sattar et al, 2004b; Dogra \& Karim, 2005), it has been shown that nurturing and fostering 'trust' in our patients improves treatment compliance, reduces relapse rates and thus helps to ameliorate their mental distress.

\section{Method}

This study was conducted at the constituent units of the South West Yorkshire Mental Health NHS Trust in Wakefield, Dewsbury, Huddersfield and Halifax. All these localities boast a high proportion of multi-ethnic and multicultural populations. We developed a postal questionnaire with eight items, some allowing dichotomous answers or a more complex choice, based on a 5-point Likert scale (from 'strongly disagree' to 'strongly agree'). This was anonymous and aimed at all the clinicians in the Trust. The questionnaire also sought information on the participants' gender, grade, ethnic origin and the country of acquiring basic medical qualification. Having obtained consent it was posted to 95 clinicians in December 2006. Data were analysed using SPSS version 10 for Windows and $\chi^{2}$-tests were used to determine whether the prescribers' responses differed with respect to their demographic details, ethnicity, level of training and place of undergraduate medical studies (UK $v$. overseas). Our study aimed to identify and assess the prescribing habits of psychiatrists that might collide with religious and cultural beliefs of patients and the doctors' opinions on the importance of informing patients when medication might contain ingredients unacceptable to them. We also sought doctors' views on the appropriate context and venue to address this issue.

\section{Results}

Out of the 95 questionnaires distributed in two mailshots, we obtained 38 responses (40\%). Most of the surveyed clinicians received their basic medical qualification from overseas medical schools; $42 \%$ were in training grades; 18 were female $(47 \%)$; for demographic information see Table 1.

The majority of respondents ( $n=27,71 \%$ ) were aware of the presence of potentially forbidden animalderived ingredients in medication and only half $(53 \%)$ considered a discussion with patients regarding the above necessary (for responses to other questions in the survey see online data supplement 1)

We provided a list of commonly used psychotropic medication to ascertain the clinicians' knowledge about whether or not they contain animal-derived gelatin and/ or stearic acid. Most of the responses were 'don't know' (Table 2). Information on the gelatin or stearic acid content of medications can be obtained from the Physicians' Desk Reference (www.pdr.net/home/ pdrHome.aspx) or electronic databases such as the Internet Drug Index (www.rxlist.com/script/main/ hp.asp).

A large majority $(n=32,84 \%)$ of respondents do not routinely inform patients of such ingredients, and only one respondent always discusses these issues with patients prior to prescribing medication. Of those respondents that 'never' discuss the ingredients, 75\% $(n=24)$ stated they would like to do that in the future. Respondents had the opportunity to add comments, particularly with regard to their reasons for providing or withholding information about medication ingredients 
Table 1. Clinician demographics

\begin{tabular}{|c|c|c|c|}
\hline \multirow[b]{2}{*}{ Parameter } & \multirow[b]{2}{*}{$\begin{array}{l}\text { Total } \\
\text { n (\%) }\end{array}$} & \multicolumn{2}{|c|}{$\begin{array}{c}\text { Medical school } \\
n(\%)\end{array}$} \\
\hline & & UK & $\begin{array}{l}\text { Inter- } \\
\text { national }\end{array}$ \\
\hline \multicolumn{4}{|l|}{ Final cohort $(n=38)$} \\
\hline Male ${ }^{1}$ & $20(53)$ & $9(24)$ & $11(29)$ \\
\hline Female & $18(47)$ & $7(18)$ & $11(29)$ \\
\hline \multicolumn{4}{|l|}{ Ethnicity } \\
\hline White $^{2}$ & $12(32)$ & $12(32)$ & \\
\hline Black and minority ethnic & $26(68)$ & $4(11)$ & $22(58)$ \\
\hline \multicolumn{4}{|l|}{ Grade } \\
\hline Consultant & $14(37)$ & $4(11)$ & $10(26)$ \\
\hline Staff Grade ${ }^{3}$ & $8(21)$ & $2(5)$ & $6(16)$ \\
\hline $\mathrm{SHO}$ & $15(40)$ & $9(24)$ & $6(16)$ \\
\hline SPR & $1(3)$ & $1(3)$ & \\
\hline \multicolumn{4}{|c|}{$P<0.001$} \\
\hline \multicolumn{4}{|c|}{ SHO, Specialist House Officer; SPR, Specialist Registrar. } \\
\hline \multicolumn{4}{|c|}{ 1. $\chi^{2}=0.145$} \\
\hline \multicolumn{4}{|l|}{ 2. $\chi^{2}=24.115$. } \\
\hline 3. $\chi^{2}=5.358$. & & & \\
\hline
\end{tabular}

Table 2. Doctors' awareness of medication containing animal derivatives

\begin{tabular}{|lccc}
\hline Medication & Aware & $\begin{array}{c}\text { Not } \\
\text { aware }\end{array}$ & $\begin{array}{c}\text { Don't } \\
\text { know }\end{array}$ \\
\hline Fluoxetine (capsules) & 7 & 1 & 30 \\
Mirtazapine (capsules) & 6 & 1 & 31 \\
Zyprexa (tablets) & 3 & 2 & 33 \\
Fluoxetine (syrup) & 2 & 5 & 31 \\
Imipramine (mixture) & 2 & 4 & 32 \\
Mirtazapine Orodispersible & 2 & 4 & 32 \\
Risperidone Quicklet & 2 & 4 & 32 \\
Venlafaxine XR & 2 & 3 & 33 \\
Imipramine (tablets) & 1 & 3 & 34 \\
Mirtazapine (tablets) & 1 & 4 & 33 \\
Risperidone & 1 & 3 & 34 \\
Venlafaxine & 1 & 4 & 33 \\
Zyprexa Velotab & 2 & 5 & 31 \\
\hline
\end{tabular}

$n=38$.

from patients in the future (see online data supplements 2 and 3). We also sought information regarding the best venue to address these issues. More than half (53\%) of the respondents felt that this discussion should only take place if and when a client identifies any concerns but a significant number (45\%) felt that it should be made part of the informed consent. Nineteen respondents (55\%) either agreed or strongly agreed with the statement that volunteering information on the presence of 'forbidden contents' of medication would negatively influence patients' future compliance with medication.

Chi-squared tests did not suggest any variables (demographic details, ethnicity, level of training or place of undergraduate medical studies) significant in clinicians' prescribing behaviour.

\section{Discussion}

To our knowledge, this is the first study in the UK of prescribers' attitudes, knowledge and opinions on the importance of informing patients when medication might contain ingredients against their beliefs, culture or religion and the potential effects on the doctor-patient relationship. Prescribing edication containing animalderived gelatin and/or stearic acid may be unavoidable in certain situations. However, people who follow certain

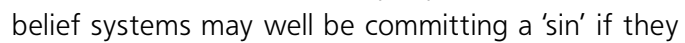
readily consume such medications. This leaves many with the daunting prospect of compromising their deeply held personal and religious values at the cost of their mental health (Sattar et al, 2004b). As practice shows, some may consciously choose to sacrifice the latter. This not only has obvious implications for patients but also for physicians who may find themselves facing ethical and potentially legal challenges. Therefore, it is imperative that full and frank discussion takes place as part of informed deliberations prior to reaching any agreement on the use of psychotropic medication. Psychiatrists should also make every effort to take a proactive approach in identifying patients that are affected by such issues and educating them on the ingredients in their prescribed medication. A basic familiarity with religious dietary restrictions (Meyer, 1988) and of available alternatives can easily fulfil this purpose.

Another approach would be for drug companies to clearly mark medication packaging with easy-to-spot ingredient labels and symbols, equivalent to the ' $V$ ' sign used on vegetarian-approved food products. This is a novel, and potentially controversial, proposal but such product label guides have been widely called for by many sections of society and have now become the accepted and expected norm for many food products. Although a number of medications (invariably modified-release, controlled-release and gastro-resistant preparations) already state the presence of gelatin in capsules, this is usually confined inconspicuously to the product information leaflet. A logical extension of this may be to clearly label such products with a single identifiable logo or mark (e.g. crossed-out ' $V$ ' sign).

Occasionally, involvement of community leaders and religious scholars from relevant faith communities in the deliberating process could not only aid understanding of these sensitive issues among healthcare professionals but might also help to instil a spirit of trust in patients. This becomes even more important, especially since the theological positions on this issue are complex. However, many faiths tend to follow the overriding principle of medical necessity and exercise leniency to preserve life (Gatrad \& Sheikh, 2000; Spitzer, 2003; Medicines Partnership Publication, 2004; Usmani, 2006). Demonstrating awareness combined with a genuine concern about how these issues impact upon patients as individuals may help to improve transparency in cases of non-compliance. Only a process such as this can facilitate a dialogue which may help patients decide on the appropriate course of their treatment. original papers 
Comprehensive policy planning on such an important issue should ideally involve both prescribing and nonprescribing healthcare professionals, pharmaceutical industry representatives, religious clerics, community leaders, the Vegetarian Society, the General Medical Council, carers and service users.

New guidance on personal beliefs and medical practice (General Medical Council, 2007) is currently being drafted (the 8-weeks' public consultation on this issue ended on 21 September 2007). It partly explores one of the aspects of our work but it specifically examines the provision of equitable access to healthcare for all people with mental health problems and potential barriers on both sides in the doctor-patient relationship. Notably, 'it is not intended to provide a comprehensive guide to religious or other beliefs and how these impact on medical care or treatment' (General Medical Council, 2007). However, we feel that further investigation into these specific aspects is also equally warranted at this time as research and guidance in this area is scarce.

\section{Limitations of the study}

Our study was limited to only one geographical area of the UK and the response rate was not particularly high. More research and empirical data are needed, particularly with regards to the views, opinions and attitudes of service users. In addition, we did not directly investigate how the cultural and religious beliefs of psychiatrists themselves might influence their prescribing habits and attitudes.

\section{Declaration of interest}

P.B. has given talks for and received hospitality from various pharmaceutical companies.

\section{Acknowledgements}

We thank Mrs Faiza Imran for her hard work and contribution in data collection and statistical analysis. We also appreciate the advice and support given by Mr Harry Smart, Mental Health Chaplain, Sheffield Care NHS Trust, and Prof. Stephen Curran and Dr Richard Marriot, South West Yorkshire Mental Health NHS Trust.

\section{References}

BATSON, C. D. \& VENTIS,W. L. (1993)

The Religious Experience: A Social,

Psychological Perspective. Oxford

University Press.

DEIN, S. (2004) Working with patients with religious beliefs. Advances in Psychiatric Treatment, 11, 287-295.

DOGRA, N. \& KARIM, K. (2005)

Diversity training for psychiatrists.

Advances in Psychiatric Treatment, 11, 159-167.

FOOD STANDARDS AGENCY (2005)

ConsumerAttitudes to Food Standards. Wave 5 (http://www.food.gov.uk/ multimedia/pdfs/casuk04.pdf)

GATRAD, A. \& SHEIKH, A. (2000) Health and disease: an Islamic framework. In: Caring for Muslim patients. Radcliffe Medical Press.

GENERAL MEDICAL COUNCIL (1998) Seeking Patient's Consent: the ethical considerations

GENERAL MEDICAL COUNCIL (2007) Personal beliefs and medical practice.

HUGHES, I. , HILL, B. \& BUDD, R. (1997)

Compliance with antipsychotic medication: from theory to practice. Journal of Mental Health, 6, 373-389.

KIROV, G., KEMP, R., KIROV, K., et al (1998) Religious faith after psychotic illness. Psychopathology, 31, 234-235.

KOENIG, H., MCCULLOUGH, M. \& LARSON, D. (2001) Handbook of Religion and Health. Oxford University Press.

LEUNG, D., DAVIES, S., WONG, S., et al (2005) Zyprexa Velotab (Olanzapine): suitable for vegetarians? Psychiatric Bulletin, 29, 275-276.

MEDICINES PARTNERSHIP PUBLICATION (2004) Informed Choice in Medicine Taking: Drugs of Porcine Origin \& Clinical Alternatives (http:// www.npc.co.uk/med.partnership/ assets/drugs-of-porcine-origin.pdf).
MEYER, M. S. (1988) Ethical principles of psychologists and religious diversity. Professional Psychology: Research and Practice, 19, 486-488.

PARGAMENT, K. \& BRANT, C. (1998) Religion and coping. In Handbook of Religion and Mental Health. California Academic Press.

PATEL, X. M. \& DAVID, S. A. (2005) Why aren't depot antipsychotic prescribed more often and what can be done about it. Advances in Psychiatric Treatment, 11, 203-213.

\section{ROYAL COLLEGE OF PSYCHIATRISTS}

(2004) College statement on covert administration of medicines. Psychiatric Bulletin, 28, 385-386.

ROBINSON, D. WOERNER, M. G., ALVIR, J. M., et al (1999) Predictors of relapse following response from a first episode of schizophrenia or schizoaffective disorder. Archives of General Psychiatry, 56, 241-247.

SATTAR, S. P., AHMED, M. S., MAJEED F. et al (2004a) Inert medication ingredients causing nonadherence due to religious beliefs. The Annals of Pharmacotherapy, 38, 621-624.

SATTAR, S. P., AHMED, M. S., MADISON, J., et al (2004b) Patient and physician attitudes to using ingredients. The Annals of Pharmacotherapy, 38, 1830-1835.

SINGSIT, S. \& NAIK, C. P. (2001) Remember the depressed vegetarian. British Journal of Psychiatry, 178, 184.

SPITZER, J. (2003) Caring for Jewish Patients. Radcliffe Medical Press.

SUBACH, R. A. \& ABDUL-EZZ, S. R. (1999) Religious reasons for discrimination of immunosuppressive medication after renal transplant. Renal Failure, 21, 223-226.

USMANI, M. M.T. (2006) The Islamic Laws of Animal Slaughter. WhiteThread Press. medications with religiously forbidden

*Waqqas Ahmad Khokhar Specialty Registrar, NorthTrent Rotational Training Scheme in Psychiatry, Waltheof Road, Sheffield S2 1PE, email: waqqaskhokhar@ doctors.org.uk, Imran Hameed Specialty Registrar, Mohammed

Mubashir Ali Specialty Registrar, NorthTrent Rotational Training Scheme in Psychiatry, Sheffield, Javaria Sadiq Clinical attaché, Mayo Hospital, Lahore, Pakistan, Peter Bowie Consultant Psychiatrist, Grenoside Grange Hospital, Saltbox Lane, Sheffield 